\title{
Correlation between the Stretching Vibrations of Aliphatic Groups and the Structural and Geochemical Properties of Crude Oils of the Same Genetic Type Using the Case of the Turija-Sever Oil Field, Pannonian Basin, Serbia
}

\author{
J. Stevanovic ${ }^{a, b, *}$, A. R. Rakitin ${ }^{a}$, and K. Stojanovic ${ }^{b}$ \\ ${ }^{a}$ Upstream Laboratory, NIS-Naftagas Science and Technology Center, Novi Sad, 21000 Serbia \\ ${ }^{b}$ Faculty of Chemistry, University of Belgrade, Belgrade, 11000 Serbia \\ *e-mail: jelena.stevanovic@nis.eu
}

\section{SUPPLEMENTARY MATERIAL}

Table A. Root-mean-square deviations (RMSD) and variances (Var.) of the peak maxima relative to mean values (Mean) for three parallel measurements of Tus-10, Tus-088, Tus-144 and Tus- 083 samples covering the entire range of the $\mathrm{CH}_{2} / \mathrm{CH}_{3}$ branching factor

\begin{tabular}{|c|c|c|c|c|c|c|c|c|c|c|c|c|c|}
\hline \multirow{2}{*}{ Sample } & \multirow{2}{*}{$\begin{array}{l}\mathrm{CH}_{2} / \\
\mathrm{CH}_{3}\end{array}$} & \multicolumn{4}{|c|}{$\mathrm{CH}_{3}$ asymmetric, $\mathrm{cm}^{-1}$} & \multicolumn{4}{|c|}{$\mathrm{CH}_{2}$ asymmetric, $\mathrm{cm}^{-1}$} & \multicolumn{4}{|c|}{$\mathrm{CH}_{2}$ symmetric, $\mathrm{cm}^{-1}$} \\
\hline & & Peak & Mean & RMSD & Var. & Peak & Mean. & RMSD & Var. & Peak & Mean. & RMSD & Var \\
\hline \multirow{3}{*}{ Tus-010 } & \multirow{3}{*}{2.61} & 2952.63 & \multirow{3}{*}{2952.72} & \multirow{3}{*}{0.14} & \multirow{3}{*}{0.02} & 2920.90 & \multirow{3}{*}{2920.76} & \multirow{3}{*}{0.28} & \multirow{3}{*}{0.08} & 2851.69 & \multirow{3}{*}{2851.59} & \multirow{3}{*}{0.22} & \multirow{3}{*}{0.05} \\
\hline & & 2952.64 & & & & 2920.44 & & & & 2851.34 & & & \\
\hline & & 2952.88 & & & & 2920.95 & & & & 2851.75 & & & \\
\hline \multirow{3}{*}{ Tus-088 } & \multirow{3}{*}{3.02} & 2952.72 & \multirow{3}{*}{2952.76} & \multirow{3}{*}{0.37} & \multirow{3}{*}{0.13} & 2919.56 & \multirow{3}{*}{2919.72} & \multirow{3}{*}{0.14} & \multirow{3}{*}{0.02} & 2850.51 & \multirow{3}{*}{2850.49} & \multirow{3}{*}{0.04} & \multirow{3}{*}{0.00} \\
\hline & & 2953.14 & & & & 2919.78 & & & & 2850.45 & & & \\
\hline & & 2952.41 & & & & 2919.81 & & & & 2850.52 & & & \\
\hline \multirow{3}{*}{ Tus-144 } & \multirow{3}{*}{3.45} & 2952.90 & \multirow{3}{*}{2953.14} & \multirow{3}{*}{0.23} & \multirow{3}{*}{0.05} & 2918.70 & \multirow{3}{*}{2918.77} & \multirow{3}{*}{0.24} & \multirow{3}{*}{0.06} & 2849.57 & & & \\
\hline & & 2953.36 & & & & 2918.57 & & & & 2849.85 & 2849.68 & 0.15 & 0.02 \\
\hline & & 2953.16 & & & & 2919.04 & & & & 2849.61 & & & \\
\hline & & 2952.85 & & & & 2917.19 & & & & 2848.98 & & & \\
\hline Tus-083 & 3.81 & 2953.47 & 2953.33 & 0.42 & 0.18 & 2917.15 & 2917.38 & 0.36 & 0.13 & 2848.82 & 2848.91 & 0.08 & 0.01 \\
\hline & & 2953.66 & & & & 2917.79 & & & & 2848.94 & & & \\
\hline
\end{tabular}

Table B. Test statistics of methylene stretching peak maxima

\begin{tabular}{|c|c|c|c|c|c|}
\hline Samples & Vibrations & $V_{1} / V_{2}$ & $\bar{s}(x)$ & $\left|\overline{x_{1}}-\overline{x_{2}}\right|$ & $\xi$ \\
\hline \multirow{2}{*}{ Tus-010 / Tus-088 } & asymmetric & $0.08 / 0.02$ & 0.224 & 1.04 & 5.69 \\
\cline { 2 - 6 } & symmetric & $0.05 / 0.00$ & 0.118 & 1.10 & 8.53 \\
\hline \multirow{2}{*}{ Tus-088 / Tus-083 } & asymmetric & $0.02 / 0.13$ & 0.274 & 2.34 & 10.46 \\
\cline { 2 - 6 } & symmetric & $0.00 / 0.01$ & 0.071 & 1.58 & 27.26 \\
\hline \multirow{2}{*}{ Tus-010/ Tus-083 } & asymmetric & $0.08 / 0.13$ & 0.324 & 3.38 & 12.78 \\
\cline { 2 - 6 } & symmetric & $0.05 / 0.01$ & 0.173 & 2.68 & 18.98 \\
\hline \multirow{2}{*}{ Tus-010 / Tus-144 } & asymmetric & $0.08 / 0.06$ & 0.265 & 1.99 & 9.20 \\
\cline { 2 - 6 } & symmetric & $0.05 / 0.02$ & 0.187 & 1.91 & 12.51 \\
\hline
\end{tabular}




\begin{tabular}{|c|c|c|c|c|c|}
\hline \multirow{2}{*}{ Tus-144 / Tus-083 } & asymmetric & $0.06 / 0.13$ & 0.308 & 1.39 & 5.53 \\
\cline { 2 - 6 } & symmetric & $0.02 / 0.01$ & 0.122 & 0.77 & 7.73 \\
\hline \multirow{2}{*}{ Tus-088 / Tus-144 } & asymmetric & $0.02 / 0.06$ & 0.200 & 0.95 & 5.82 \\
\cline { 2 - 6 } & symmetric & $0.00 / 0.02$ & 0.100 & 0.81 & 9.92 \\
\hline
\end{tabular}

$\bar{s}(x)=\sqrt{\frac{f_{1} V_{1}+f_{2} V_{2}}{f_{1}+f_{z}}}-$ RMSD of two series of measurements;

$\overline{\bar{x}_{1}}-\overline{x_{2}} \mid \sqrt{\frac{n_{1} \cdot n_{2}}{n_{1}+n_{2}}}$

$\xi=\overline{\bar{s}(\chi)} \sqrt{\frac{n_{1}+n_{2}}{2}}-$ test statistics;

$n_{1}$ and $n_{2}$ - number of parallel measurements (3) for the calculation of $\overline{x_{1}}$ and $\overline{x_{\mathbf{z}}}$ means;

$f_{1}$ и $f_{2}$ - degrees of freedom of series of measurements $(n-1)$;

$V_{1}$ и $V_{2}$ - variances of series of measurements;

The limiting value of $\xi$ for $P=0.9$ confidence probability is 2.92 . 